\title{
Mn12Ac inhibits the migration, invasion and epithelial-mesenchymal transition of lung cancer cells by downregulating the Wnt/ $\beta$-catenin and PI3K/AKT signaling pathways
}

\author{
ZIHAO CHEN ${ }^{1,2}$, JIANGBO HE $^{3}$, XIQIAN XING ${ }^{4},{\text { PING } \text { LI }^{3}, \text { WEI ZHANG }}^{5}$, ZHUXIU TONG $^{5}$, \\ XIAOJIE JING ${ }^{5}$, LICHENG LI ${ }^{3}$, DIAN LIU ${ }^{3}$, QIONG WU ${ }^{6}$ and HONGPING JU ${ }^{3,7}$
}

${ }^{1}$ Gruaduate School of Hebei Medical University, Shijiazhuang, Hebei 050017; ${ }^{2}$ The Third Department of Surgery,

The Fourth Hospital of Hebei Medical University, Shijiazhuang, Hebei 050011; ${ }^{3}$ School of Medicine, Kunming University, Kunming, Yunnan 650214; ${ }^{4}$ First Department of Respiratory Medicine, Yan'an Hospital Affiliated to Kunming Medical University, Kunming, Yunnan 650051; ${ }^{5}$ Department of Medicine, The People's Hospital of Economic and Technological Development Zone, Kunming, Yunnan 650000; ${ }^{6}$ Department of Chemical Science and Technology, Kunming University, Kunming, Yunnan 650214;

${ }^{7}$ Respiratory System Disease Prevention and Control of Public Service Platform of Science and Technology in Yunnan Province, Kunming, Yunnan 650000, P.R. China

Received September 6, 2017; Accepted June 13, 2018

DOI: $10.3892 / \mathrm{ol} .2018 .9136$

\begin{abstract}
Lung cancer is the leading cause of global cancer-associated mortality, therefore it is important to reveal the molecular mechanisms of lung cancer progression and to develop novel therapeutic targets. The results of the present study identified that manganese-12 acetate (Mn12Ac) was able to significantly inhibit the migration and invasion of A549 cells. Western blotting demonstrated that treatment with Mn12Ac was able to upregulate E-cadherin, and downregulate $\mathrm{N}$-cadherin and vimentin. It was also identified by a quantitative polymerase chain reaction analysis that $\mathrm{Mn} 12 \mathrm{Ac}$ was able to reduce the mRNA expression levels of EMT-associated transcription factors Snail, Slug, Twist-related protein 1 and zinc finger E-box-binding homeobox 1. It was also demonstrated that Mn12Ac was able to reduce the expression levels of Wnt and $\beta$-catenin proteins, and suppress the phosphorylation of phosphoinositide 3-kinase (PI3K) and AKT in A549 cells. Notably, it was revealed that Mn12Ac was able to decrease the mRNA and protein expression
\end{abstract}

Correspondence to: Professor Hongping Ju, School of Medicine, Kunming University, 2 Puxin Road, Kunming, Yunnan 650214, P.R. China

E-mail: juhongping@yeah.net

Professor Qiong Wu, Department of Chemical Science and Technology, Kunming University, 2 Puxin Road, Kunming, Yunnan 650214, P.R. China

E-mail: wuqiong_chem@yeah.net

Keywords: manganese-12 acetate, migration, epithelial-mesenchymal transition, Wnt, phosphoinositide 3-kinase levels of programmed death ligand-1. Taken together, the results suggested that Mn12Ac is able to inhibit cell migration, invasion and EMT in lung cancer cells by regulating the Wnt/ $\beta$-catenin and PI3K/AKT signaling pathways.

\section{Introduction}

Lung cancer is the leading cause of cancer mortality, and there were $\sim 1,800,000$ incident lung cancer cases in 2012 globally, representing $\sim 13 \%$ of the total global cancer incidence (1). Lung cancer primarily consists of two histological types: Small cell lung cancer and non-small-cell lung cancer (NSCLC), which account for 15 and $85 \%$ of total cases of lung cancer, respectively (2). Lung adenocarcinoma (LAC), which is the most widespread histological type of NSCLC, causes $>500,000$ mortalities every year worldwide (3). Therefore, it is important to reveal the molecular mechanisms of LAC progression and development, and to develop novel therapeutic targets for patients with LAC.

Epithelial-mesenchymal transition (EMT) is an important process that is characterized by the loss of cell polarity and cell-cell contact, and is activated in tumor metastasis and resistance to chemoradiotherapy $(4,5)$. Activation of EMT is associated with the altered expression of numerous genes, including the downregulation of epithelial markers such as occluden-1 and E-cadherin, and the upregulation of mesenchymal markers including $\mathrm{N}$-cadherin and vimentin (6). A number of studies have demonstrated that EMT is a critical process in tumor invasion and metastasis in NSCLC (7-9).

Manganese-12 acetate (Mn12Ac) is a magnetically bistable molecule exhibiting a combination of strong magnetic anisotropy and high spin properties $(10,11)$. However, there have been no studies concerning the association between Mn12Ac and disease therapy, particularly in cancer. In the present study, the 
biological function of Mn12Ac in lung cancer treatment was investigated.

\section{Materials and methods}

Cell culture. The human lung cancer A549 cell line (American Type Culture Collection, Manassas, VA, USA) was cultured in RPMI-1640 medium (Invitrogen; Thermo Fisher Scientific, Inc., Waltham, MA, USA) with $10 \%$ fetal bovine serum (GE Healthcare, Chicago, IL, USA), $100 \mathrm{U} / \mathrm{ml}$ penicillin and $100 \mathrm{~g} / \mathrm{ml}$ streptomycin at $37^{\circ} \mathrm{C}$ in a humidified incubator containing $5 \% \mathrm{CO}_{2}$. Cells were treated with $100 \mathrm{nM} \mathrm{Mn12 \textrm {Ac }}$ (dissolved in $\mathrm{H}_{2} \mathrm{O}$, and $\mathrm{H}_{2} \mathrm{O}$ was used as control) and cultured at $37^{\circ} \mathrm{C}$ for $48 \mathrm{~h}$, and subsequent experiments were performed.

Total RNA extraction and quantitative polymerase chain reaction ( $q P C R)$ assay. Total RNA was isolated from cancer cells using the RNeasy Mini kit according to the protocol of the manufacturer (Qiagen GmbH, Hilden, Germany) and used for the qPCR assay. Three independent experimental repeats of qPCR were performed.

qPCR was used to detect the mRNA expression of E-cadherin, N-cadherin, vimentin, zinc finger protein SNAI1 (Snail), zinc finger protein SNAI2 (Slug), twist-related protein 1 (Twist1), zinc finger E-box-binding homeobox 1 (ZEB1) and programmed death-ligand 1 (PD-L1). The PCR reactions were performed in a total volume of $20 \mu \mathrm{l}$, including $10 \mu \mathrm{l} 2 \mathrm{X}$ Power SYBR $^{\circledR}$ Green PCR Master Mix (Applied Biosystems; Thermo Fisher Scientific, Inc., Waltham, MA, USA), $2 \mu 1$ cDNA (5 ng/ $\mu \mathrm{l})$ and $1 \mu \mathrm{l}$ primer mix (10 $\mu \mathrm{M}$ each). PCR amplification and detection were performed in a LightCycler 480 II (Roche Applied Science, Penzberg, Germany) as follows: Initial denaturation at $95^{\circ} \mathrm{C}$ for $10 \mathrm{~min}$, then 40 cycles of denaturation at $95^{\circ} \mathrm{C}$ for $15 \mathrm{sec}$ and annealing and elongation at $60^{\circ} \mathrm{C}$ for $1 \mathrm{~min}$. The relative gene expression was calculated using the comparative $\mathrm{Cq}$ method. The gene expression of the target gene was normalized to an endogenous reference gene (GAPDH), and data relative to the calibrator were calculated using the formula $2^{-\Delta \Delta \mathrm{Cq}} . \Delta \mathrm{Cq}$ was calculated by subtracting the average GAPDH $\mathrm{Cq}$ value from the average $\mathrm{Cq}$ value of the gene of interest (12). The ratio defined the level of relative expression of the target gene to that of GAPDH. The primers were as follows: E-cadherin forward primer, 5'-GAACGCATTGCCACATAC AC-3'; reverse primer, 5'-GAATTCGGGCTTGTTGTCAT-3'; N-cadherin forward primer, 5'-GTGCCATTAGCCAAGGGA ATTCAGC-3', reverse primer, 5'-GCGTTCCTGTTCCAC TCATAGGAGG-3'; vimentin forward primer, 5'-TGAGTA CCGGAGACAGGTCGAG-3', reverse primer, 5'-TAGCAG CTTCAACGCAAAGTTC-3'; Snail forward primer, 5'-ACC ACTATGCCGCGCTCTT-3', reverse primer, 5'-GGTCGT AGGGCTGCTGGAA-3'; Slug forward primer, 5'-GCGCAT GCTCCATTGTCTTAC-3', reverse primer, 5'-AGGCACTTG GAAGGGGTATTG-3'; Twist1 forward primer, 5'-AGAAGT CTGCGGGCTGTGGCG-3', reverse primer, 5'-GAGGGC AGCGTGGGGATGATC-3'; ZEB1 forward primer, 5'-CTA CTCAACTACGGTCAGCCC-3', reverse primer, 5'-TTGGGC GGTGTAGAATCAGAG-3'; programmed death ligand 1 (PD-L1) forward primer, 5'-GAACTACCTCTGGCACAT СCT-3', reverse primer, 5'-CACATCCATCATTCTCCC TTT-3'; GAPDH forward primer, 5'-AAATCCCATCACCAT
CTTCCAG-3' and reverse primer, 5'-GAGTCCTTCCACGAT ACCAAAGTTG-3'.

Western blotting. The cells were lysed in the lysis buffer $(20 \mathrm{mM}$ Tris, 2 mM EDTA, 50 mM 2-mecaptoethanol, 10\% glycerol, $\mathrm{pH}$ 7.4). The homogenates were placed on ice for $30 \mathrm{~min}$ and centrifuged at $12,000 \mathrm{x}$ g for $15 \mathrm{~min}$ at $4^{\circ} \mathrm{C}$. Subsequently, the protein concentration of the lysates was determined using a Protein Assay kit (Bio-Rad Laboratories, Inc., Hercules, CA, USA). Equal amounts (15 $\mu \mathrm{g})$ of total proteins were loaded onto a 10\% PAGE, electrophoretically transferred to polyvinylidene difluoride membrane, and then blocked with $10 \%$ non-fat milk for $2 \mathrm{~h}$ at room temperature. The membranes were incubated with specific primary antibodies overnight at $4{ }^{\circ} \mathrm{C}$ and probed with corresponding secondary antibodies (goat anti-rabbit IgG-HRP, sc-2004, 1:5,000 and goat anti-mouse IgG-HRP, sc-2005, 1:5,000, Santa Cruz Biotechnology, Inc., Dallas, TX, USA) for $1 \mathrm{~h}$ at room temperature. The protein bands were visualized using Enhanced Chemiluminescence Blotting Detection reagents (Applygen Technologies, Inc., Beijing, China). The primary antibodies used are as follows: E-cadherin (1:1,000; sc-71009; Santa Cruz Biotechnology, Inc.), N-cadherin (1:1,000; sc-59987; Santa Cruz Biotechnology, Inc.), vimentin (1:1,000; sc-80975; Santa Cruz Biotechnology, Inc.), Wnt1 (1:1,000; sc-6266; Santa Cruz Biotechnology, Inc.), $\beta$-catenin (1:1,000; sc-65480; Santa Cruz Biotechnology, Inc.), phosphorylated-phosphoinositide 3-kinase (p-PI3K; 1:1,000; 4228; Cell Signaling Technology, Inc., Danvers, MA, USA), PI3K (1:1,000; sc-376112; Santa Cruz Biotechnology, Inc.), p-AKT (1:1,000; sc-271964; Santa Cruz Biotechnology, Inc.), AKT (1:1,000; sc-1619; Santa Cruz Biotechnology, Inc.), PD-L1 $(1: 1,000 ; 13684$; Cell Signaling Technology, Inc.) and $\beta$-actin (1:5,000; sc-517582; Santa Cruz Biotechnology, Inc.).

Transwell assay. For the Transwell invasion assay, $60 \mu 1$ Matrige ${ }^{\circledR}$ was diluted with precooled serum-free medium in a ratio of 1:4, and was added to the bottom of the Transwell chamber and incubated for $1 \mathrm{~h}$ at $37^{\circ} \mathrm{C}$. A total of $2 \times 10^{5}$ cells ( $\mathrm{Mn} 12 \mathrm{Ac}$ treated cells and the $\mathrm{H}_{2} \mathrm{O}$ treated control cells) were seeded into the upper chambers (24-well insert; pore size $8 \mu \mathrm{m}$ ), while medium supplemented with $10 \%$ FBS $(600 \mu 1)$ was placed in the lower chamber. Following incubation at $37^{\circ} \mathrm{C}$ for $24 \mathrm{~h}$, the cells on the upper chamber side of the inserts were removed gently with a cotton swab. The inserts were then fixed with $4 \%$ methanol for $15 \mathrm{~min}$ at room temperature and stained with $0.1 \%$ crystal violet for $15 \mathrm{~min}$ at room temperature. The mean number of migratory cells was calculated by counting five randomly selected fields each time under the light microscope at x 200 magnification. The experiments were repeated three times.

For the Transwell migration assay, the remaining protocol was the same as the Transwell invasion assay, except that the inserts were not pre-coated with Matrigel ${ }^{\circledR}$.

Statistical analysis. All data were analyzed by unpaired Student's t-test or one-way analysis of variance with post hoc comparisons with Tukey's honest significant difference test for multiple group comparisons using SPSS version 20.0 (IBM Corp., Armonk, NY, USA) and GraphPad Prism (version 5.0; GraphPad, Inc., La Jolla, CA, USA). P<0.05 was considered to indicate a statistically significant difference. 
A
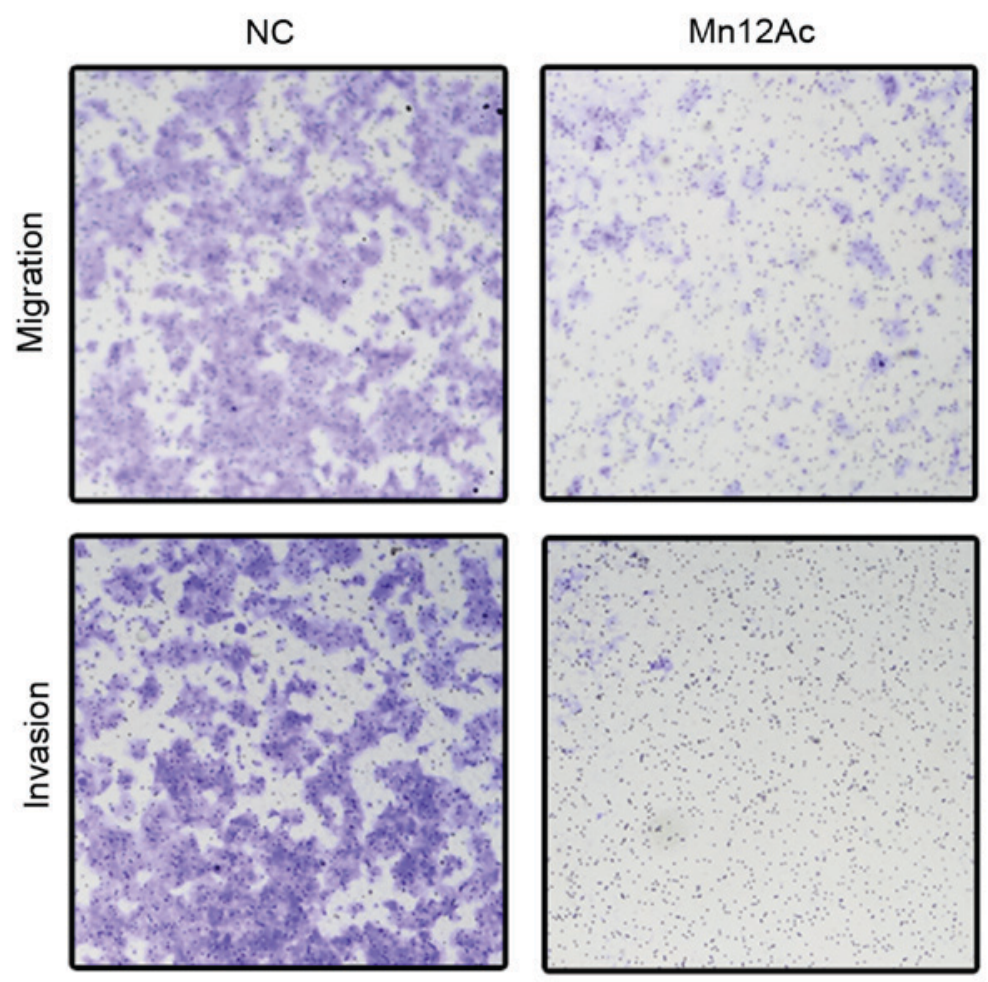

B
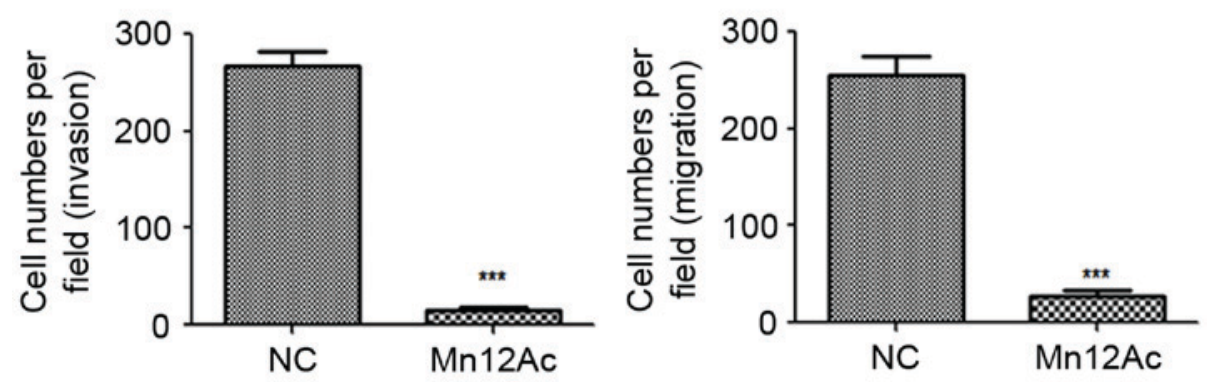

Figure 1. Mn12Ac inhibits the migration and invasion of A549 lung cancer cells. (A) Transwell assay analysis of the migratory and invasive abilities of lung cancer cells with a light microscope at x200 magnification. (B) Statistical analysis of the results from the Transwell migration and invasion assays. ${ }^{* * *} \mathrm{P}<0.001$ vs. negative group. Mn12Ac, manganese-12 acetate; $\mathrm{NC}$, negative control.

\section{Results}

Mn12Ac is able to significantly inhibit the migration and invasion of A549 lung cancer cells. In order to investigate the anti-tumor activity of Mn12Ac, Transwell migration and invasion assays were performed to analyze the effects of Mn12Ac on lung cancer cell metastasis. The results demonstrated that Mn12Ac was able to significantly inhibit the migration and invasion of A549 cells compared with the negative control (Fig. 1A and B).

Mn12Ac inhibits EMT in A549 lung cancer cells. EMT is activated during tumor metastasis and serves an important role in the process (13); therefore whether Mn12Ac affected the EMT process in A549 lung cancer cells was investigated. The qPCR assay results indicated that $\mathrm{Mn} 12 \mathrm{Ac}$ was able to significantly upregulate the epithelial marker E-cadherin, and downregulate the mesenchymal markers $\mathrm{N}$-cadherin and vimentin in A549 cells (Fig. 2A). In addition, it was indicated by western blotting that $\mathrm{Mn} 12 \mathrm{Ac}$ was also able to markedly upregulate the protein expression of E-cadherin and reduce the protein expression levels of N-cadherin and vimentin (Fig. 2B).

Mn12Ac significantly inhibits the expressions of EMT-associated transcription factors in A549 lung cancer cells. Snail, slug, twist1 and ZEB1 are EMT-associated transcription factors in cancer (14-16). Therefore, whether Mn12Ac regulated EMT via affecting these transcription factors was examined. Using qPCR, it was revealed that Mn12Ac was able to significantly decrease the mRNA expression levels of Snail, Slug, Twist1 and ZEB1 in A549 cancer cells (Fig. 3). These results suggested that Mn12Ac inhibited EMT in lung cancer cells by downregulating EMT-inducing transcription factors.

Mn12Ac inhibits the Wnt/ $\beta$-catenin and PI3K/AKT signaling pathways. The Wnt/ $\beta$-catenin and PI3K/AKT signaling pathways serve important roles in the regulation of EMT (17-19), and it was hypothesized that Mn12Ac may inhibit EMT in lung cancer cells by regulating these two pathways. Using western blotting assay, it was identified that Mn12Ac was 
A
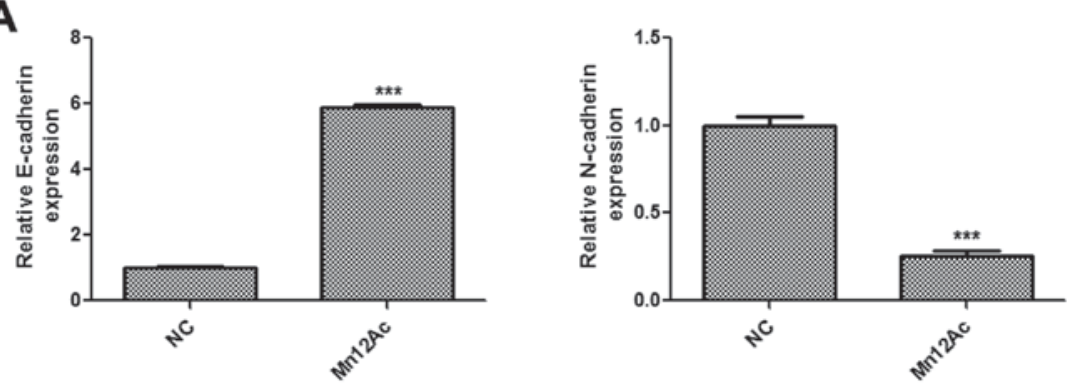

B
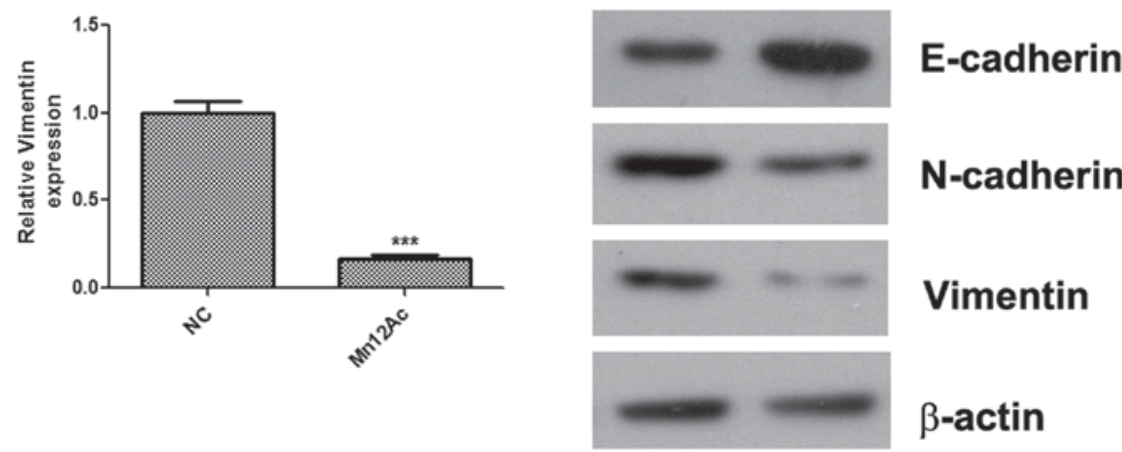

Figure 2. Mn12Ac inhibits EMT in lung cancer cells. (A) mRNA expressions of EMT markers as detected using quantitative polymerase chain reaction analysis. (B) Protein expression of EMT markers as detected using western blotting assay. ${ }^{* * *} \mathrm{P}<0.001$ vs. negative control. Mn12Ac, manganese-12 acetate; NC, negative control; EMT, epithelial mesenchymal transition; E-cadherin, epithelial cadherin; N-cadherin, neural cadherin.
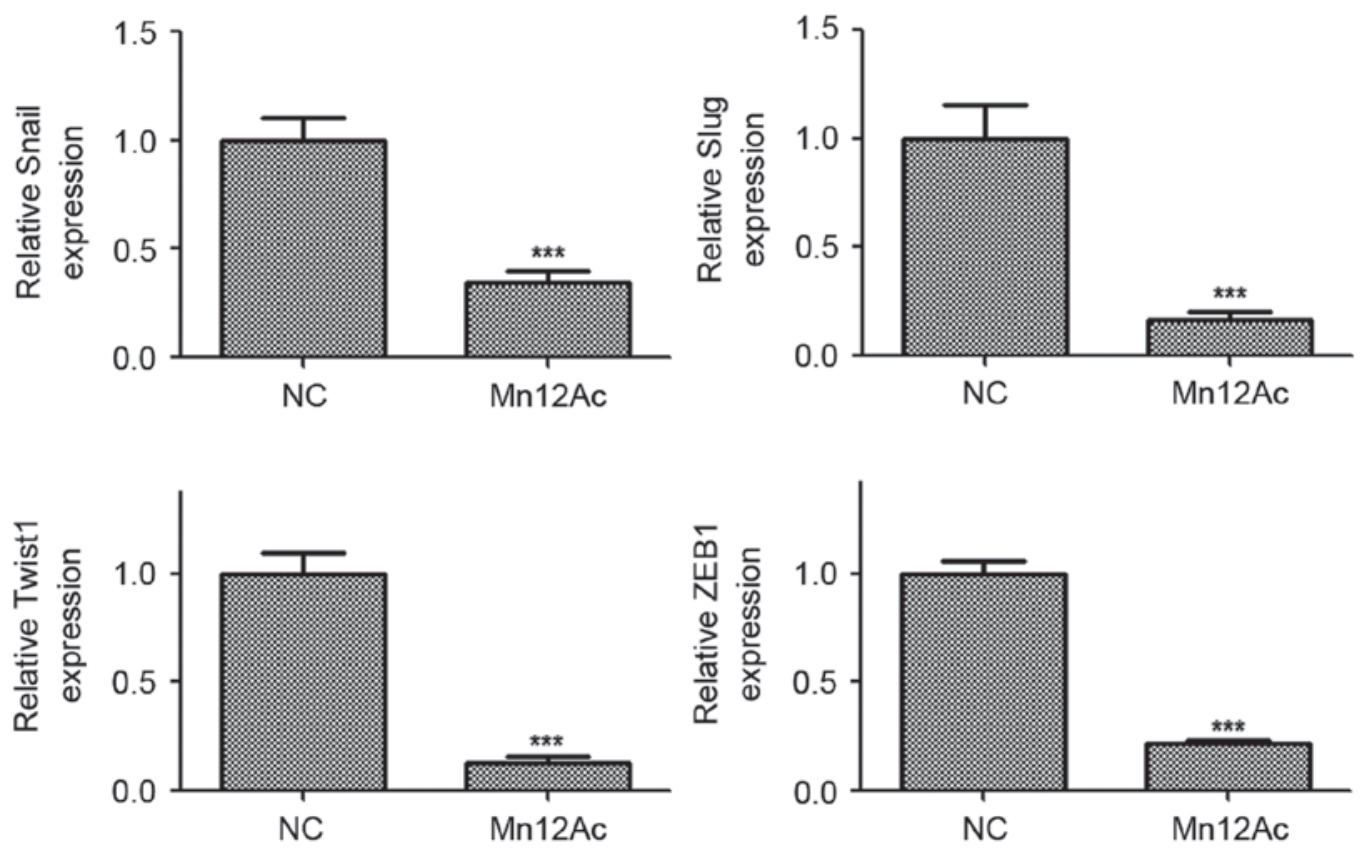

Figure 3. Mn12Ac decreases the expression levels of EMT-associated transcription factors. ${ }^{* * *} \mathrm{P}<0.001$ vs. negative group. Mn12Ac, manganese-12 acetate; NC, negative control; EMT, epithelial mesenchymal transition; Twist1, twist-related protein 1; ZEB1, zinc finger E-box-binding homeobox 1.

able to markedly reduce the expression levels of Wnt1 and $\beta$-catenin and inhibit the phosphorylation of PI3K and AKT in A549 lung cancer cells (Fig. 4).

Mn12Ac decreases the $m R N A$ and protein expression levels of PD-L1. PD-1/PD-L1-targeted immunotherapy has emerged as a promising therapeutic strategy for lung cancer, and PD-L1 expression was associated with EMT transition in lung cancer (20). Therefore, the present study investigated whether Mn12Ac affected PD-L1 in lung cancer. Notably, the results indicated that Mn12Ac was able to reduce the mRNA and protein expression levels of PD-L1 in A549 lung cancer cells by qPCR and western blotting assay, respectively (Fig. 5). 


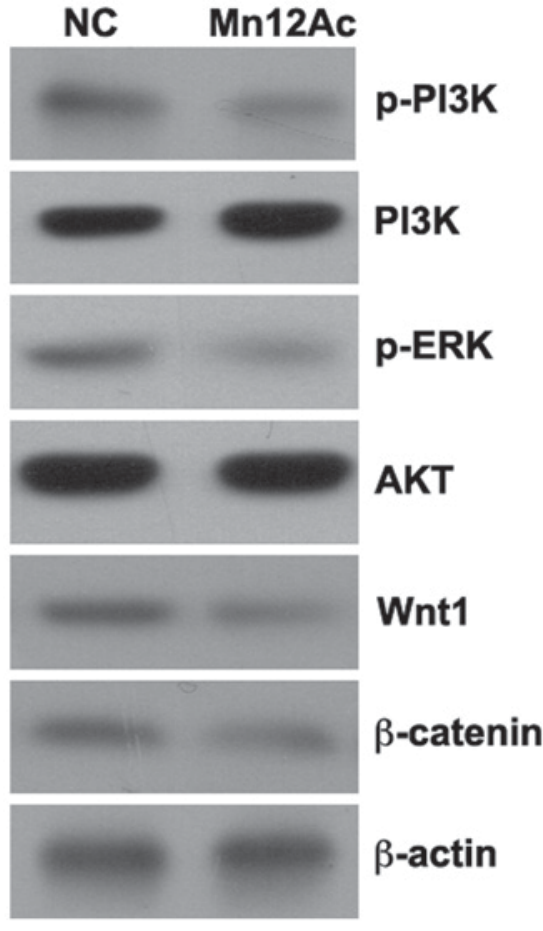

Figure 4. Mn12Ac inhibits the PI3K/AKT and Wnt/ $\beta$-catenin signaling pathways. The expressions of Wnt 1 and $\beta$-catenin and the phosphorylation of PI3K and AKT were assessed by western blot analysis. Mn12Ac, manganese-12 acetate; NC, negative control.

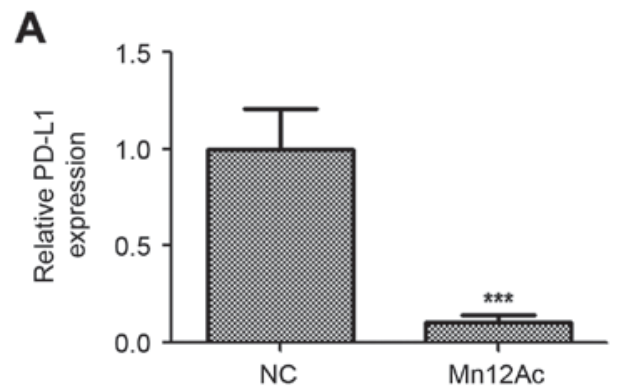

B

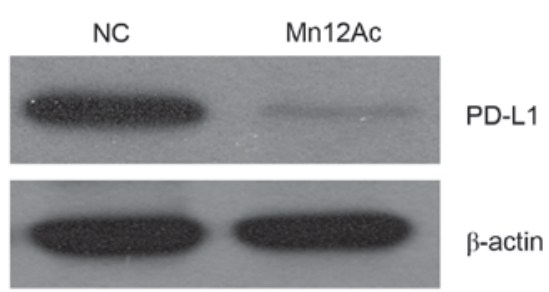

Figure 5. Mn12Ac decreases the PD-L1 expression in A549 lung cancer cells (A) The mRNA expression of PD-L1 was assessed by quantitative polymerase chain reaction analysis. (B) Protein expression of PD-L1 was assessed by western blotting assay. ${ }^{* * *} \mathrm{P}<0.001$ vs. negative control. Mn12Ac, manganese-12 acetate; NC, negative control; PD-L1, programmed death-ligand 1.

\section{Discussion}

Human lung cancer is the most common cause of global cancer-associated mortality (21). Lung cancer develops through a multistep process with oncogenic mutations in lung epithelial cells (22). It is important to reveal the molecular mechanisms of LAC progression and development, and to develop novel therapeutic targets for patients with LAC.

Metastasis is the primary cause of cancer-associated mortality, and the epithelial EMT process is critical for epithelial cell cancer progression. The results of the present study demonstrated that Mn12Ac was able to significantly inhibit the migration and invasion of A549 lung cancer cells. These results suggested that Mn12Ac suppressed the EMT process in lung cancer cells. EMT is a morphological change in cells from an epithelial form to a fibroblast-like mesenchymal form. In addition to these morphological changes, key biomarkers that involved in the steps of the process include cell adhesion molecules such as E-cadherin, $\mathrm{N}$-cadherin, and transcription factors Snail, Slug, Twist1 and ZEB1 $(5,23)$. The expression levels of these EMT molecules were demonstrated to be associated with drug resistance, and the inhibition of EMT may interfere with tumor progression and drug resistance (24). The results of the present study suggested that Mn12Ac may inhibit the EMT process by downregulating the transcription factors Snail, Slug, Twist1 and ZEB1. In addition, the results of the present study suggest that Mn12Ac may promote drug sensitivity in cancer therapy.

The PI3K/AKT signaling pathway serves an important role in the EMT process and tumor metastasis. Shao et al (25) suggested that irisin may inhibit EMT and the invasion of lung cancer cells by regulating the PI3K/AKT/Snail signaling pathway. Tumor protein D52 inhibited the growth and metastasis of renal cell carcinoma cells by downregulating the levels of p-PI3K and p-Akt (26). Curcumin was also reported to suppress EMT in renal tubular epithelial cells through the inhibition of the Akt/mTOR signaling pathway (19). The Wnt/ $\beta$-catenin signaling pathway was also associated with the EMT process. CDGSH iron sulfur domain 2 may promote proliferation and EMT in pancreatic cancer cells by upregulating the Wnt/ $\beta$-catenin signaling pathway (27). Wang et al (28) reported that calpain small subunit 1 promoted EMT in human melanoma cells through the activation of the Wnt/ $\beta$-catenin signaling pathway (28). Zuo et al (29) observed that long non-coding RNA protein SPRY4 intronic transcript 1 was able to modulate trophoblast cell invasion, migration and EMT processes by regulating the Wnt/ $\beta$-catenin signaling pathway (29). The results of the present study revealed that Mn12Ac may inhibit the $\mathrm{Wnt} / \beta$-catenin and PI3K/AKT signaling pathways in lung cancer cells.

PD-L1 in tumor cells is known to promote immune escape of cancer cells by interacting with programmed cell death protein 1 in tumor-infiltrating immune cells (30). Immunotherapy targeting these molecules is emerging as a novel strategy for the treatment of different types of cancer. Notably, the results of the present study indicated that Mn12Ac was able to markedly reduce the expression of PD-L1 at the level of mRNA and protein in lung cancer cells.

Taken together, the results of the present study revealed that Mn12Ac may significantly inhibit migration, invasion and EMT in lung cancer cells by suppressing the Wnt/ $\beta$-catenin and PI3K/AKT signaling pathways, and Mn12Ac may also reduce the expression of $\mathrm{PD}-\mathrm{L} 1$. In the future, additional studies should focus on the potential clinical applications of Mn12Ac. 


\section{Acknowledgements}

Not applicable.

\section{Funding}

The present study was supported by the Key Project of Kunming Technology Program (grant no. 2015-1-S-00877, 2015-1-S-00576).

\section{Availability of data and materials}

The datasets used and/or analyzed during the present study are available from the corresponding author.

\section{Author's contributions}

CZ, WQ and JH designed the work that led to the submission. HJ and XX performed RT-qPCR analysis. LP, ZW, TZ, JX, LL and LD conducted western blot analysis. $\mathrm{CZ}$ and $\mathrm{JH}$ drafted the manuscript.

\section{Ethics approval and consent to participate}

Not applicable.

\section{Patient consent for publication}

Not applicable.

\section{Competing interests}

The authors declare that they have no competing interests.

\section{References}

1. Torre LA, Bray F, Siegel RL, Ferlay J, Lortet-Tieulent J and Jemal A: Global cancer statistics, 2012. CA Cancer J Clin 65: 87-108, 2015.

2. Herbst RS, Heymach JV and Lippman SM: Lung cancer. N Engl J Med 359: 1367-1380, 2008.

3. Imielinski M, Berger AH, Hammerman PS, Hernandez B, Pugh TJ, Hodis E, Cho J, Suh J, Capelletti M, Sivachenko A, et al: Mapping the hallmarks of lung adenocarcinoma with massively parallel sequencing. Cell 150: 1107-1120, 2012.

4. Smith BN and Bhowmick NA: Role of EMT in metastasis and therapy resistance. J Clin Med 5: pii: E17, 2016.

5. Lamouille S, Xu J and Derynck R: Molecular mechanisms of epithelial-mesenchymal transition. Nat Rev Mol Cell Biol 15: 178-196, 2014.

6. Seton-Rogers S: Epithelial-mesenchymal transition: Untangling EMT's functions. Nat Rev Cancer 16: 1, 2016.

7. Wang DX, Zou YJ, Zhuang XB, Chen SX, Lin Y, Li WL, Lin JJ and Lin ZQ: Sulforaphane suppresses EMT and metastasis in human lung cancer through miR-616-5p-mediated GSK3 $\beta$ / $\beta$-catenin signaling pathways. Acta Pharmacol Sin 38: 241-251, 2017.

8. Ye Z, Yin S, Su Z, Bai M, Zhang H, Hei Z and Cai S: Downregulation of miR-101 contributes to epithelial-mesenchymal transition in cisplatin resistance of NSCLC cells by targeting ROCK2. Oncotarget 7: 37524-37535, 2016.

9. Chen S, Jiao S, Jia Y and Li Y: Effects of targeted silencing of FOXC1 gene on proliferation and in vitro migration of human non-small-cell lung carcinoma cells. Am J Transl Res 8: 3309-3318, 2016

10. Rakvin B, Zilić D, North JM and Dalal NS: Probing magnetic fields on crystals of the nanomagnet Mn12-acetate by electron paramagnetic resonance. J Magn Reson 165: 260-264, 2003.
11. Morello A, Bakharev ON, Brom HB, Sessoli R and de Jongh LJ: Nuclear spin dynamics in the quantum regime of a single-molecule magnet. Phys Rev Lett 93: 197202, 2004.

12. Livak KJ and Schmittgen TD: Analysis of relative gene expression data using real-time quantitative PCR and the $2^{-\Delta \Delta C_{\mathrm{T}}}$ method. Methods 25: 402-408, 2001.

13. Guo C, Zhao D, Zhang Q, Liu S and Sun MZ: miR-429 suppresses tumor migration and invasion by targeting CRKL in hepatocellular carcinoma via inhibiting Raf/MEK/ERK pathway and epithelial-mesenchymal transition. Sci Rep 8: 2375, 2018.

14. Amoroso MR, Matassa DS, Agliarulo I, Avolio R, Lu H, Sisinni L, Lettini G, Gabra H, Landriscina $M$ and Esposito F: TRAP1 downregulation in human ovarian cancer enhances invasion and epithelial-mesenchymal transition. Cell Death Dis 7: e2522, 2016.

15. Liang W, Lai Y, Zhu M, Huang S, Feng W and Gu X: Combretastatin A4 regulates proliferation, migration, invasion, and apoptosis of thyroid cancer cells via PI3K/Akt signaling pathway. Med Sci Monit 22: 4911-4917, 2016.

16. Hammers H, Fu C, Gerber S, Berg SVD, Steenwinkel F, Moriarty W, Keizman D, Kachhap S and Carducci MA: Epithelial-mesenchymal transition: A mechanism of resistance to VEGF pathway inhibition in genitourinary cancers. J Clin Oncol 30 (5_suppl): S390, 2012.

17. Liu MH, Fu WJ, Cui YH, Guo QN and Zhou Y: Downregulation of Semaphorin-3F is associated with poor prognostic significance in osteosarcoma patients. Am J Cancer Res 6: 2252-2262, 2016.

18. Liu B, Pan CF, He ZC, Wang J, Wang PL, Ma T, Xia Y and Chen YJ: Long noncoding RNA-LET suppresses tumor growth and EMT in lung adenocarcinoma. Biomed Res Int 2016: 4693471, 2016.

19. Zhu FQ, Chen MJ, Zhu M, Zhao RS, Qiu W, Xu X, Liu H, Zhao HW, Yu RJ, Wu XF, et al: Curcumin suppresses epithelial-mesenchymal transition of renal tubular epithelial cells through the inhibition of Akt/mTOR pathway. Biol Pharm Bull 40: 17-24, 2017.

20. Kim S, Koh J, Kim MY, Kwon D, Go H, Kim YA, Jeon YK and Chung DH: PD-L1 expression is associated with epithelial-to-mesenchymal transition in adenocarcinoma of the lung. Hum Pathol 58: 7-14, 2016.

21. Siegel R, Naishadham D and Jemal A: Cancer statistics, 2012. CA Cancer J Clin 62: 10-29, 2012.

22. Larsen JE and Minna JD: Molecular biology of lung cancer: Clinical implications. Clin Chest Med 32: 703-740, 2011.

23. Arai K, Eguchi T, Rahman MM, Sakamoto R, Masuda N, Nakatsura T, Calderwood SK, Kozaki K and Itoh M: A novel high-throughput 3D screening system for EMT inhibitors: A pilot screening discovered the EMT inhibitory activity of CDK2 inhibitor SU9516. PLoS One 11: e0162394, 2016.

24. Dopeso H, Jiao HK, Cuesta AM, Henze AT, Jurida L, Kracht M, Acker-Palmer A, Garvalov BK and Acker T: PHD3 controls lung cancer metastasis and resistance to EGFR inhibitors through TGFo. Cancer Res 78: 1805-1819, 2018.

25. Shao L, Li H, Chen J, Song H, Zhang Y, Wu F, Wang W, Zhang W, Wang F, Li H and Tang D: Irisin suppresses the migration, proliferation, and invasion of lung cancer cells via inhibition of epithelial-to-mesenchymal transition. Biochem Biophys Res Commun 485: 598-605, 2017.

26. Zhao Z, Liu H, Hou J, Li T, Du X, Zhao X, Xu W, Xu W and Chang J: Tumor protein D52 (TPD52) inhibits growth and metastasis in renal cell carcinoma cells through the PI3K/Akt signaling pathway. Oncol Res 25: 773-779, 2017.

27. Yang Y, Bai YS and Wang Q: CDGSH iron sulfur domain 2 activates proliferation and EMT of pancreatic cancer cells via Wnt $/ \beta$-catenin pathway and has prognostic value in human pancreatic cancer. Oncol Res 25: 605-615, 2017.

28. Wang E, Wang D, Li B, Ma H, Wang C, Guan L, Zhang H, Yi L and Li S: Capn4 promotes epithelial-mesenchymal transition in human melanoma cells through activation of the Wnt $/ \beta$-catenin pathway. Oncol Rep 37: 379-387, 2017.

29. Zuo Q, Huang S, Zou Y, Xu Y, Jiang Z, Zou S, Xu H and Sun L: The Lnc RNA SPRY4-IT1 modulates trophoblast cell invasion and migration by affecting the epithelial-mesenchymal transition. Sci Rep 6: 37183, 2016.

30. Chatterjee J, Dai W, Aziz NHA, Teo PY, Wahba J, Phelps DL, Maine CJ, Whilding LM, Dina R, Trevisan G, et al: Clinical use of programmed cell death-1 and its ligand expression as discriminatory and predictive markers in ovarian cancer. Clin Cancer Res 23: 3453-3460, 2017.

This work is licensed under a Creative Commons Attribution-NonCommercial-NoDerivatives 4.0 International (CC BY-NC-ND 4.0) License. 